\title{
Revisiting Acid-Fast Bacilli Microscopy of Concentrated Sputum Smears as an Efficient Tool for the Diagnosis of Tuberculosis: A Study from a Tertiary Care Centre in Southern India
}

\author{
J. Anto Jesuraj Uday Kumar ${ }^{1,2 *}$, Chirag Dhar ${ }^{1,3}$, Hiresave Srinivasa ${ }^{2}$ \\ ${ }^{1}$ Division of Infectious Diseases, St. John's Research Institute, Bangalore, India \\ ${ }^{2}$ Department of Microbiology, St. John's Medical College and Hospital, Bangalore, India \\ ${ }^{3}$ Department of Pulmonary Medicine, St. John's Medical College and Hospital, Bangalore, India \\ Email: *antouday@gmail.com
}

How to cite this paper: Kumar, J.A.J.U., Dhar, C. and Srinivasa, H. (2017) Revisiting Acid-Fast Bacilli Microscopy of Concentrated Sputum Smears as an Efficient Tool for the Diagnosis of Tuberculosis: A Study from a Tertiary Care Centre in Southern India. Journal of Tuberculosis Research, $\mathbf{5}$, 146-154.

https://doi.org/10.4236/jtr.2017.52016

Received: March 16, 2017

Accepted: June 25, 2017

Published: June 29, 2017

Copyright $\odot 2017$ by authors and Scientific Research Publishing Inc. This work is licensed under the Creative Commons Attribution International License (CC BY 4.0).

http://creativecommons.org/licenses/by/4.0/

\section{(c) (i) Open Access}

\begin{abstract}
Background and objectives: With 2.2 million new cases every year, Tuberculosis (TB) continues to be an epidemic of large proportions in India. Conventional direct sputum smear microscopy, though limited in its sensitivity, is still the most common method of testing for TB. Newer techniques such as concentrated sputum microscopy, have shown some promise in improving this limited sensitivity. We have compared the efficacy of concentrated sputum versus the direct smear technique in 1000 sputum samples of patients suspected to be suffering from TB. Methods: A total of 1000 sputum specimens were collected for direct acid-fast bacilli (AFB) smear, concentrated AFB smear and culture from St. John's Medical College and Hospital. 39 contaminated samples were (3.9\%) omitted during the final analysis. Mycobacterial culture was used as the reference standard method for the detection of TB. Results: 184 and 198 of the 961 samples were found to AFB positive by direct smear microscopy and concentrated smear technique respectively. The measured sensitivity and specificity of direct smear microscopy were $69.86 \%$ and $95.82 \%$, while that of concentrated smear microscopy was $76.71 \%$ and $95.96 \%$ respectively. 33 samples found to be negative by the direct smear method turned out to be positive by the concentrated smear technique. Conclusions: Though our study suggests no significant statistical difference between the two techniques of detecting pulmonary tuberculosis, we recommend the use of the concentrated technique in centres such as ours, where facilities are already in place. In this way, the number of cases of TB that remain untreated may significantly come down.
\end{abstract}




\section{Keywords}

AFB, TB Diagnosis, Concentrated Smear, Direct Smear, ZN Staining

\section{Introduction}

Tuberculosis continues to be an epidemic of large proportions in India. Every year, 2.2 million new cases are detected. TB also accounts for more than 478,000 deaths each year. In 2015, there were an estimated 10.4 million cases globally and nearly 1.4 million deaths. These figures make TB the second largest cause of death among patients with infectious diseases [1]. A landmark paper by Nirmalan et. al suggests that the number of TB cases in India may be much larger than earlier estimated. This is due to the vast number of cases that are treated by the private sector [2].

Early and efficient diagnosis of TB is thus, absolutely necessary. Presently, the gold standard for diagnosing TB is sputum culture. This method, though, requires culture facilities and increases the turn-around time for diagnosis. Due to these limitations, Direct Ziehl-Neelson microscopy for the detection of Acid Fast Bacilli remains the most common point-of-care diagnostic test across India. This technique, which has been in use for more than 130 years now, is believed to be the fastest way to detect TB in developing countries [3] [4]. Apart from its fast turn-around times, ZN staining is both simple and inexpensive. Yet, its biggest drawback is its sensitivity. Even under optimal conditions, this technique can diagnose only $60 \%$ of the sputum culture positive TB cases [5] and requires $10^{4}$ to $10^{5}$ bacilli per $\mathrm{ml}$ of sputum for its success [6]. Newer diagnostic tests such as GeneXpert have been developed in an attempt to better this sensitivity.

Since the turn of the millennium, there has been growing evidence that liquefaction of sputum and subsequent concentration by centrifugation and sedimentation followed by acid fast staining can significantly increase the efficacy of microscopy in TB detection [7]. N-acetyl-L-cysteine (NALC) along with 4\% Sodium Hydroxide $(\mathrm{NaOH})$ is most commonly used for sputum preparation. On the downside, this preparation of concentrated sputum requires trained personnel, higher biosafety levels and increased time taken to make a diagnosis.

In the quest to develop a robust diagnostic test for our tertiary care hospital, we revisited this technique of concentrated sputum microscopy. We have compared the efficacy of concentrated sputum versus the direct smear technique in 1000 sputum samples of patients suspected to be suffering from TB. Additionally, we have also cultured these sputum samples to measure the sensitivity of these two microscopy-based techniques.

\section{Methodology}

The study was approved by the Ethical Review Committee of St. John's Medical College and Hospital, Bangalore, India. All study subjects were enrolled in the 
study only after they had provided informed written consent.

The study design is a comparative study of diagnostic accuracy. The concentrated smear and Reference standard (Direct Smear) tests were done simultaneously. The study involved 1000 patients with suspected tuberculosis-both in-patients and out-patients who presented themselves to a tertiary-care teaching hospital in urban south India. Sputum samples of patients aged 4 years and above with suspected tuberculosis, who were registered for sputum AFB smear examination in the RNTCP Clinic, were included in this study. The samples were collected from the RNTCP clinic between January 2013 to December 2015, purely based on the inclusion in registry and convenience sampling. Sputum samples which were less than $1 \mathrm{ml}$ were excluded. Patient information was anonymized prior to analysis.

Collected samples were transported in a spill-proof box to the laboratory for AFB microscopy and culture.

A loop full of the purulent part of the sputum was used for the direct smears. The remaining sputum was then processed by the NALC-NaOH digestion-decontamination method [8]. Equal volumes of $4 \% \mathrm{NaOH}$ and $2.94 \%$ sodium citrate containing $0.5 \%$ NALC were added to each tube and the contents within were vortexed and incubated at $25^{\circ} \mathrm{C}$ for 15 minutes. At regular intervals, the tubes were shaken. Phosphate-buffered saline $(\mathrm{pH}$ 6.8) was added at the end of the incubation period and volume was made up to $45 \mathrm{ml}$. The tube was then centrifuged at $3000 \mathrm{X} g$ for 15 minutes. The supernatant was poured off and the resulting sediments were re-suspended in $1-2 \mathrm{ml}$ of phosphate-buffered saline. This suspension was used to prepare an additional smear (concentrated smear) [9].

2 - 3 drops of re-suspended sediments were also inoculated on LowensteinJensen (L-J) culture media. $500 \mu \mathrm{l}$ of this re-suspended sediments was also inoculated in a Mycobacterium growth indicator tube (MGIT) along with PNTA (a combination of antimicrobial drugs). Both these tubes were incubated at $37^{\circ} \mathrm{C}$ for 8 weeks and 6 weeks respectively or until growth was noticed on the solid medium and MGIT tubes indicated growth as per the manufacturer's instructions [10] [11] [12]. All contaminated tubes were discarded and the corresponding primary samples were not included in the final analysis. The growth in liquid cultures was confirmed by microscopy. Samples found to be positive using Micro MGIT reader were also streaked on blood agar to rule out contamination.

Both direct and concentrated smears were stained by the Ziehl-Neelsen's technique. The slides were read within 48 hours of preparation using a light microscope (magnification 1000x). Smears, where AFB were noticed, were graded as per the RNTCP guidelines: 1 to 9 AFB seen in 100 microscopic fields scored as scanty positive, 10 to 99 AFB seen in 100 fields scored as $1+, 1$ to 10 AFB seen per field in at least 50 fields scored as $2+$, more than 10 AFB seen per field in at least 20 fields scored as 3+ [13]. Direct smears and concentrated smears were examined separately and blinded to prevent observer bias. 


\section{Statistical Analysis}

Direct smear and concentrated smear were independently compared with sputum culture as the benchmark for TB diagnosis. The sensitivity, specificity, positive predictive value and negative predictive value were calculated using a $2 \times 2$ table. Further, McNemar's test (GraphPad, an online software) was used for calculation of statistical significance.

\section{Results}

Out of the 1000 specimens collected for the study, 961 were included in the final analysis. The remaining 39 sputum samples were contaminated and were not included. 184 (19.1\%) of these 961 samples were found to be AFB positive and the remaining $80.9 \%$ were found to be AFB negative by direct AFB smear. The following were the different grades of the positive specimens, 24 (13.0\%) were scanty positive, $39(21.2 \%)$ were $1+, 71(38.6 \%)$ were $2+$ and $50(27.1 \%)$ were $3+$. In contrast, $198(20.6 \%)$ were found to be AFB positive by the concentrated smear technique. Among the positive specimens 29 (14.6\%), 45 (22.7\%), 45 (22.7\%), 79 (39.9\%) were scanty positive, $1+, 2+$ and $3+$ respectively.

Further, we compared direct smear and concentrated smear with sputum culture as the gold standard of TB diagnosis. By sputum culture, 219 (22.7\%) samples were positive. Table 1 represents the comparison of direct smear versus sputum culture.

The sensitivity and specificity of direct smear as compared to sputum culture was $69.86 \%$ and $95.82 \%$ respectively. The positive predictive value and negative predictive value were found to be $83.15 \%$ and $91.51 \%$ respectively. $\mathrm{P}$ value calculated was found to be 0.0006 (Table 2).

In contrast, the sensitivity and specificity of concentrated smear as compared to sputum culture was $76.71 \%$ and $95.96 \%$ respectively. The positive predictive value and negative predictive value were found to be $84.85 \%$ and $93.32 \%$ respectively (P-value of 0.02).

Table 1. Concentrated smear versus sputum culture for the detection of AFB.

\begin{tabular}{ccc}
\hline & Culture positive (219) & Culture negative (742) \\
\hline Smear Positive (198) & 168 & 30 \\
Smear Negative (763) & 51 & 712
\end{tabular}

Note: Out of the 1000 sputum samples included in the study, 39 samples were contaminated and were excluded during the final analysis.

Table 2. Direct smear versus sputum culture for the detection of AFB.

\begin{tabular}{ccc}
\hline & Culture positive (219) & Culture negative (742) \\
\hline Smear Positive (184) & 153 & 31 \\
Smear Negative (777) & 66 & 711 \\
\hline
\end{tabular}

Note: Out of the 1000 sputum samples included in the study, 39 samples were contaminated and were excluded during the final analysis. 


\section{Discussion}

This study was intended to evaluate the utility of concentrated smear as an alternative to direct smear for the detection of AFB in sputum samples. The difference in sensitivities of direct and concentrated smear was found to be statistically insignificant $(69.86 \%$ and $76.71 \%$ respectively). Our study also showed negligible difference in the specificity of the two tests $(95.82 \%$ by direct smear and 95.96\% by concentrated smear). A comparison of direct smear and concentrated smear technique, independent of culture results, yielded a p-value which was also statistically insignificant.

Multiple studies have been conducted in the past to ascertain the utility of concentrated smear versus direct smear. A study conducted by Barez, et al. [14] showed that the sensitivity was nearly the same by both methods: $81.6 \%$ for the direct method and $82.7 \%$ for the concentrated method. Similarly, Cattamanchi et al. [15] were unable to find any difference between the direct and concentrated sputum smear microscopy methods. They have reported a sensitivity of $51 \%$ and $52 \%$ for direct and concentrated smear microscopy respectively.

There have been other studies that suggest the concentrated smear technique is better than the older direct smear technique. One of these is a study conducted in Bangladesh (TB endemic country) by Uddin et al. [16] which concurs with this opinion and reports a sensitivity of $83 \%$ by the concentrated smear technique as opposed to $71 \%$ by the direct smear. Another one of these is a study by Peterson et al. conducted [17] in two differing laboratory set-ups (a tertiary care hospital laboratory and multiple other outpatient clinics) found that in the tertiary care hospital arm of the study, the direct smear was considerably less sensitive than the concentrated sputum smear ( $28 \%$ and $51 \%$, respectively).

The statistical analysis of our results suggests that the efficacy of concentrated smear microscopy is not significantly superior to the direct smear microscopy technique. Yet, with the unyielding increase in the number of TB cases in India, the concentrated smear microscopy technique may significantly increase our ability to detect TB efficiently. Additionally, the concentrated sputum when smeared on a slide spreads evenly and provides a clearer light microscopy image to the observer.

Interestingly, 33 samples in our study were negative by direct smear microscopy, but positive by the concentrated smear technique. Despite the statistical insignificance, our analysis suggests that this additional detection capability of concentrated smear is of paramount importance. Of these 33, 19 were found to culture positive. The results of these 33 samples on concentrated smear is detailed in Table 3. On the other hand, 19 samples were found to be negative on concentrated smear technique but positive by direct smear. Yet, 14 of these 19 were culture negative. This may have occurred due to improper collection, concentration and preparation of samples, inadequate staining, or due to inappropriate microscopic observations. Further studies must be conducted to ascertain if this is explained exclusively by human error.

Few of the sputum specimens were positive by both the direct and concen- 
Table 3. Distribution of 33 samples that were negative for AFB by Direct Smear technique but positive by concentrated smear technique.

\begin{tabular}{cc}
\hline Concentrated smear grade & Number \\
\hline Scanty & 21 \\
$1+$ & 11 \\
$2+$ & 1 \\
$3+$ & 0 \\
Total & 33 \\
\hline
\end{tabular}

trated smear microscopy yet negative by culture. The clinical data suggests that these patients were on anti-TB medication at the time of sputum collection. During the course of treatment, the killed or dead bacilli remain in the respiratory tract (sputum) and are likely to be detected by microscopy but not in culture.

There is growing evidence to suggest that the concentrated method is better than the direct method. This method, though, is difficult to implement in ancillary TB laboratories of developing countries due to the following constraints: limitations of irregular power supply that affect centrifugation and refrigeration of reagents; limited financial resources; lack of skilled workforce; inadequate training capacity and inadequate biosafety arrangements [16]. Further, aerosolization of the TB bacilli during centrifugation, vortexing and handling of concentrated sputum samples poses a threat to healthcare workers in these laboratories. Also, the sterility of these reagents must be ensured. Thus, the requirement of an autoclave facility is an absolute must.

In a TB endemic country like India, most of the Primary health care units and secondary health care units use direct smear microscopy for the diagnosis of TB with low sensitivity. However, as per the results of our study, it may be suggested that concentrated smear microscopy is used in place of conventional direct microscopy in centres with trained personnel, power back up and autoclave facilities. This will help in identifying a higher number of true positive cases at an early stage. This, translated to the national level could significantly aid in combatting the TB epidemic. Alternately, concentrated smear microscopy may be used on sputum samples that turn out to be negative by the direct smear technique. This would increase the sensitivity without compromising on the established method of detection of TB. It must be noted here, that this concentrated smear technique is probably not the best alternative to the established direct smear microscopy method, given its higher cost and resource requirement.

Newer techniques such as the LED fluorescent microscopy have shown some promise with higher sensitivity rates as compared to the conventional ZN staining and light microscopy. LED FM provides a brighter background making it easier for technicians to identify slides with fewer bacilli [18]. Since the middle of 2012, 200 Indian medical colleges have been instructed to use the LED-FM technique instead of the older ZN staining method [19]. A study conducted by 
Badri et al. [20] showed that this technique is better at picking up 'presumptive MDR-TB cases' at the time of follow-up sputum collection. Further studies must be carried out to identify the microscopy techniques that are ideal for TB detection.

\section{Conclusions}

In conclusion, despite finding no statistical advantage of concentrated smear microscopy technique over direct smear microscopy in the detection of TB, we recommend the use of the concentrated smear technique where facilities are available. The increase in sensitivity may appear to be marginal but could significantly decrease the number of $\mathrm{TB}$ cases that go undetected.

\section{Summary at a Glance}

This study was conducted to revisit the concentrated sputum smear microscopy technique, and assess its efficacy in the detection of pulmonary tuberculosis. Statistically, no significant difference was found between the concentrated smear technique and the conventional direct smear microscopy. Yet, we suggest the use of the concentrated technique in centres with the appropriate facilities.

\section{Acknowledgements}

We would like to acknowledge Dr George D'Souza, Dr. Justy Antony Chiramal and Sumithra Selvam for their valuable time and contribution to this study. We also acknowledge St John's Medical College \& Hospital RNTCP clinic as well as the Department of Microbiology for their support in patient recruitment and data collection. We would also like to acknowledge the St John's Research Institute for giving us the laboratory support and funding for the tests and material involved.

\section{Financial Support}

This project was funded exclusively by the Division of Infectious Diseases Unit of the St John's Research Institute where the study was conducted.

\section{References}

[1] WHO (2016) Global Tuberculosis Report 2016.

[2] Arinaminpathy, N., Batra, D., Khaparde, S., Vualnam, T., Maheshwari, N., Sharma, L., et al. (2016) The Number of Privately Treated Tuberculosis Cases in India: An Estimation from Drug Sales Data. The Lancet Infectious Diseases, 16, 1255-1260.

[3] Aber, V.R., Allen, B.W., Mitchison, D.A., Ayuma, P., Edwards, E.A. and Keyes, A.B. (1980) Quality Control in Tuberculosis Bacteriology. 1. Laboratory Studies on Isolated Positive Cultures and the Efficiency of Direct Smear Examination. Tubercle, 61, 123-133.

[4] Bird, B.R., Denniston, M.M., Huebner, R.E. and Good, R.C. (1996) Changing Practices in Mycobacteriology: A Follow-Up Survey of State and Territorial Public Health Laboratories. Journal of Clinical Microbiology, 34, 554-559.

[5] World Health Organization (1193) WHO Declares TB a Global Emergency. Health 
Horizon. http://www.who.int/tb/publications/global_report/en/

[6] Tattersfield, A. (2005) Toman's Tuberculosis: Case Detection, Treatment and Monitoring: Questions and Answers, 2nd Edition. Occupational and Environmental Medicine, 62, 70 .

[7] Ängeby, K.A.K., Alvarado-Gálvez, C., Pineda-García, L. and Hoffner, S.E. (2000) Improved Sputum Microscopy for a More Sensitive Diagnosis of Pulmonary Tuberculosis. Technical Note. The International Journal of Tuberculosis and Lung Disease, 4, 684-687.

[8] Betty, A., Forbes, D.F.S. and WeissFeld, A.S. (2007) Bailey \& Scott's Diagnostic Microbiology. 12th Edition, Elsevier, Amsterdam.

[9] BACTEC MGIT 960 System User's Manual 2004/06. Becton, Dickinson and Company, Sparks.

[10] Cornfield, D.B., Beavis, K.G., Greene, J.A., Bojak, M. and Bondi, J. (1997) Mycobacterial Growth and Bacterial Contamination in the Mycobacteria Growth Indicator Tube and BACTEC 460 Culture Systems. Journal of Clinical Microbiology, 35, 2068-2071.

[11] Pfyffer, G.E., Welscher, H.M., Kissling, P., Cieslak, C., Casal, M.J., Gutierrez, J., et al. (1997) Comparison of the Mycobacteria Growth Indicator Tube (MGIT) with Radiometric and Solid Culture for Recovery of Acid-Fast Bacilli. Journal of Clinical Microbiology, 35, 364-368.

[12] Leitritz, L., Schubert, S., Bucherl, B., Masch, A., Heesemann, J. and Roggenkamp, A. (2001) Evaluation of BACTEC MGIT 960 and BACTEC 460TB Systems for Recovery of Mycobacteria from Clinical Specimens of a University Hospital with Low Incidence of Tuberculosis. Journal of Clinical Microbiology, 39, 3764-3767. https://doi.org/10.1128/JCM.39.10.3764-3767.2001

[13] RNTCP (2010) Status TB Report. Directorate General of Health Services, Ministry of Health and Family Welfare, New Delhi.

[14] Marie Yvette, C.B.M., Myrna, T., Mendoza, M.D., Regina, S., Celada, R.M.T., Heidi, R. and Santos, R.M.T. (1995) Accuracy of AFB in Relation to TB Culture in Detection of Pulmonary Tuberculosis. Journal of Microbiology and Infectious Diseases, 24.

[15] Cattamanchi, A., Dowdy, D.W., Davis, J.L., Worodria, W., Yoo, S., Joloba, M., et al. (2009) Sensitivity of Direct versus Concentrated Sputum Smear Microscopy in HIV-Infected Patients Suspected of Having Pulmonary Tuberculosis. BMC Infectious Diseases, 9, 53. https://doi.org/10.1186/1471-2334-9-53

[16] Uddin, M.K., Chowdhury, M.R., Ahmed, S., Rahman, M.T., Khatun, R., van Leth, F., et al. (2013) Comparison of Direct versus Concentrated Smear Microscopy in Detection of Pulmonary Tuberculosis. BMC Research Notes, 6, 291.

https://doi.org/10.1186/1756-0500-6-291

[17] Peterson, E.M., Nakasone, A., Platon-DeLeon, J.M., Jang, Y., de La Maza, L.M. and Desmond, E. (1999) Comparison of Direct and Concentrated Acid-Fast Smears to Identify Specimens Culture Positive for Mycobacterium spp. Journal of Clinical Microbiology, 37, 3564-3568.

[18] Central Tuberculosis Division (2005) Revised National Tuberculosis Control Program Technical and Operational Guidelines for Tuberculosis Control. Directorate General of Health Services, Ministry of Health and Family Welfare, Government of India, New Delhi.

[19] Reza, L.W., Satyanarayana, S., Pandey, A., Kumar, S., Devendrappa, N.M., Anand, L., et al. (2013) LED Fluorescence Microscopy Increases the Detection of Smear- 
Positive Pulmonary Tuberculosis in Medical Colleges of India. Public Health Action, 3, 240-242. https://doi.org/10.5588/pha.13.0021

[20] Thapa, B., Reza, L.W., Kumar, A.M., Pandey, A., Satyanarayana, S. and Chadha, S. (2015) Light Emitting Diode Fluorescence Microscopy Increased the Detection of Smear-Positives during Follow-Up of Tuberculosis Patients in India: Program Implications. BMC Research Notes, 8, 596. https://doi.org/10.1186/s13104-015-1584-z

\section{Scientific Research Publishing}

Submit or recommend next manuscript to SCIRP and we will provide best service for you:

Accepting pre-submission inquiries through Email, Facebook, LinkedIn, Twitter, etc. A wide selection of journals (inclusive of 9 subjects, more than 200 journals)

Providing 24-hour high-quality service

User-friendly online submission system

Fair and swift peer-review system

Efficient typesetting and proofreading procedure

Display of the result of downloads and visits, as well as the number of cited articles

Maximum dissemination of your research work

Submit your manuscript at: http://papersubmission.scirp.org/

Or contact jtr@scirp.org 